\title{
KUTAMBUWA UGONJUWA: CONCEPTS OF ILLNESS AND TRANSFORMATION AMONG THE TABWA OF ZAIRE
}

\author{
Christopher Davis-Roberts \\ Department of Anthropology, Center for Afro-American and African Studies, \\ University of Michigan, U.S.A.
}

\begin{abstract}
Beginning with the description of a single instance of problematic illness, this paper moves toward the comprehension of Tabwa therapeutics by placing them in their most complete conceptual context. Decisions made regarding methods of treatment. the loci of therapy (both physical and social). and the means of assessing therapeutic effectiveness reflect an epistemology which includes the overlapping domains of physiology, diagnosis, divination and religious and magical practices; domains whose different contents are nevertheless tightly interwoven by a relatively small number of underlying principles. The articulation of these principles makes clear that Tabwa concepts of illness and transformation form a coherent whole within which both traditional and European medicines take their appropriate and complementary places.
\end{abstract}

In Tabwaland, as elsewhere, the medical system exists and is experienced not as the discursive, analytic prose of the specialist or the observer, but rather as the narrative or saga of specific illness occurrences, episodes which cut to varying depths across the normal flow of life. For this reason, it is perhaps appropriate that we begin our study of Tabwa medical thought with the telling of one such tale. Embodied within it are the concepts of process, of materiality and of transformation which underlie not only the medical system, but also the definition of reality itself. Through an examination of these, we will be able to accurately locate within the epistemologic system the therapeutic alternatives from among which BaTabwa regularly choose [1].

\section{KAPLTA'S DAUGHTER'S ILLVESS}

Early in 1977, Malaika, the three-year old daughter of a close friend, Kaputa, developed two fluctuating swellings on the back of her head. These first emitted pus, then developed lesions which would not heal. During the course of the succeeding months Malaika's parents, her extended family, and my husband and I applied all the therapeutic means at our collective disposal in an attempt to effect cure. Creams, ointments, and penicillin injections were alternated with the application of powdered plant substances and herbal washes, but to no avail.

By the end of six months time, Kaputa and Kalwa. his wife, were beginning to ask themselves what sort of illness could be so resistant to treatment with both traditional ( $\mathrm{a}$ a nchi) and European (va Kizungu) medicines (dawa). Though they felt they should seek divinatory insight into the cause of their daughter's affliction, they postponed doing so because the illness was not life-threatening and they had many other social and financial obligations.

In June, 1977, I acquired some capsules of tetracycline, and, with these, again attempted treatment of Malaika's illness. After five days, the pus and swelling stopped; and though the medication ran out shortly after this, by the end of the month the child's lesions had closed.
At about this same time, however, Malaika began to have other symptoms. Every morning upon awaking, she would begin to scratch, and swellings would appear all over her body; sometimes especially affecting her eyes and face. Though the swellings would disappear after an hour or so, the itching would remain, a sequence of events characteristic of the illness masoli ('urticaria' or 'hives' [2]). IN addition. Malaika began suffering nocturnal fevers which were so severe that they caused mild convulsions (kustukastuka) and prompted her parents to sit up all night (kukesha) watching over her.

Though the fever would remit during the day, the child's temperature was never normal, and my initial efforts to treat the fever with chloroquine were only partially successful. This limited response, coupled with the presence of the hives and the character of the fever led me to eliminate malaria as a possible diagnosis and to instead suspect that Malaika had contracted bilharziasis when visiting her mother's natal village in the mountains [3]. My diagnosis was all but useless, however, for the dispensary was without medicines, and I myself had none of the niridazole required for treatment.

On July 6, 1977, a message was sent to Kaputa while he was at our house, to the effect that Malaika had just been stricken with 'convulsions' (ndege) while playing at home. What he found when he got there was that his daughter had suffered a series of particularly violent shudderings or tiny seizures which had been controlled on the spot by his mother, who had used the traditional medical treatment of throwing herbal infusions over the child with a broom (kumusampula). Against the background of Malaika's ongoing nightly fevers, this incident precipitated Kaputa's decision to seek divination immediately, and also to search the village for itinerant traders who might have niridazolc to sell or to exchange for dried fish.

By the next day, both problems had been fortunately resolved. In the afternoon, Kaputa had located people down from Kalemie who were selling niridazole at a price which, though expensive, was still within his reach. He bought ten tablets, and began 
administering them to his daughter that night, following the dosage indicated by my physician's manual.

In the evening, Kaputa decided upon a diviner and sent him the "arrow' necessary to begin the process of divination. In choosing a diviner, he employed at least two significant evaluative criteria. First, he selected the type of divination (Tulunga) considered to be the most accurate and the most likely to reveal deadly etiologic agents such as avenging ghosts and sorcerers. Second, he selected a practitioner to whom he was unknown and who was himself relatively new to the village of Mpala. In this way, Kaputa structured the situation so as to increase the veracity of the diviner's insights into his case, for, as he told me, numerous seances with the same diviner or seances with a person who knows one tend to result in the repeated emergence of a few problems, while the real causes of the illness remain unknown.

The 'arrow' (mshale) by which the divination was begun was a small coin (a one-likuta piece). which had been placed on the ground and prepared by invocations made first by Kaputa, then by Kalwa. In their statements, each of the pair had cited all possible causes of illness (adultery, thievery, breach of familial obligations, quarrels with neighbors, etc.), and instructed that the cause, whatever it might be, present itself to the diviner for the sake of the child's health. Though such invocations have a standardized structure, their statements were nevertheless direct reflections of the many and complex relationships of which Kaputa and Kalwa were part, each of which might have been the precipitant of their daughter's affliction. After its preparation, the 'arrow' was given to the diviner, who placed it at the head of his bed and, during the next two nights dreamt of the cause of Malaika's illness.

On the morning of July 9, 1977, Kaputa, Kalwa and I went to the diviner's together. As is customary with tulunga divination, a pot of freshly-drawn water was prepared and set to boil, and when it was hot, a lukusu seed was dropped into it by the diviner. For some time the practitioner completely ignored us and concentrated on the oracular device. He silently questioned it, intently scrutinizing the steaming pot as he shook his rattles; then plunging his hand into the boiling water and retricving the seed each time the oracle's answer was no. Finally, he gave us a parable and then a pronouncement which ended in a question.

He said that in his dream he had seen a woman whose husband had died. She had not been inherited by a successor, but another man had nevertheless entered the house. Then a third man had come, the two men had fought, and the woman was also beaten. Kaputa and Kalwa were to apply this to themselves and their families and to determine who these people might be.

After some discussion the young couple revealed that the features presented by the diviner corresponded to the situation of Kalwa's mother. When Kalwa's own father had died, her mother had been given a successor, as is the custom (bupvani) in Tabwaland. However, when this man died, his lineage group had refused to provide the widow with another, saying that she was destroying their line. Others claimed that the husband had been killed by an avenging ghost (kibanda) deriving from his family's own evil deeds, and that they were abusing Kalwa's mother in an effort to conceal the truth from the outside world. Eventually, the widow was inherited by a man from another, related lineage group; but, as Kaputa put it, he came "like a thief in the night", performed the necessary ceremony and then left. leaving the widow improperly cleansed.

Shortly after this, Kalwa's mother began to have episodes of 'lunacy' (wazimu). These were attributed to attacks upon her by the avenging ghost made under the mistaken impression that she was still attached to her deceased husband. Her children sought redress of grievance from their paternal kinsmen in court, but were denied it because they lacked sufficient political weight in the village. Privately the judges told them that they had justice on their side though they could not be openly granted it. During the succecding months, Kalwa's mother's condition worsened, until she finally became incurably mad. Harmless, she wandered from village to village, sometimes living with Kaputa and Kalwa, sometimes staying with others, and sometimes sleeping out of doors, naked and isolated from the intercourse of reason.

With the completion of the progress of their mother's illness, rupture in relations between Kalwa's group of siblings, their lineage group, and their affines was such that juridical resolution became impossible The only release from the avenging ghost was therefore to be sought in the ceremony called "throwing the person in the bush" (kumutupa mutu mupori). In this, the diviner would mediate between the afflicted group and the etiologic agent responsible for their sufferings. Much of the short discussion after the diviner's pronouncement was devoted to consideration of the details of this therapeutic alternative, and to the ways in which it might be carried out.

After the seance concluded, I spoke with Kaputa about the diviner's hypothesis, and found him to be in essential agreement with what the practitioner had said. Such a result had been obtained years before after the death of his first child. At the time, Kalwa's step-father had admitted to having an avenging ghost, but had refused to do anything about it. Since then, however, other problems had intervened, and had been repeatedly cited by local diviners as the cause of illness in Kaputa's house.

Kaputa and Kalwa thought to consult with her siblings, to go with them to another diviner, and, if the same result was obtained, to undertake the ceremony. This, however, was going to take a minimum of some weeks. In the meantime, the effect of the pronouncement itself, were it accurate, would be the remission of Malaika's symptoms. When Kaputa returned to our house that evening, he brought with him the news that Kalwa had arrived home to find Malaika playing, and with her skin cool for the first time in weeks.

For presentation here, this episode has been denuded of much of its detail. Nevertheless, in this, the skeleton of its barest structure, are visible many of the principles that shall be explored below. In his hypothesis, the diviner brought together into one explanatory whole may different domains or levels of experience. These included the nature of the illness itself, both as physiologic symptoms and as an event occurring in the life of a three-year-old child. Included 
as well were the personal history of that child's parents and grandparents; and a category of etiologic agent which contains implicit within itself a prognosis and a non-bodily therafy.

This interweaving of illness, history and etiology is more than the simple coordination or correspondence of somewhat disparate factors. Rather, the varied domains are tightly interconnected by an epistemology which constructs/explains reality on the basis of certain significant premises. Among those are:

(a) the transposition of a single process from one experiential domain to another (e.g. physiologic, social, physical, verbal, etc.);

(b) an emphasis upon the unique event as that which has special communicative power, and the concomitant privilege accorded to the witness whose understanding unites with clarity what other's blindness leaves unconnected;

(c) a relation between hidden processes and manifest appearances such that the latter can be construed as artifacts of the former, artifacts which are, nonetheless, the only means by which these processes can be known; and finally

(d) the concept of time as developmental process and ideas specifying a complementarity of relations among words, events and objects in such a processual reality.

Each of these can be examined more completely in relation to a different aspect of the Tabwa medical system as a whole.

\section{CONCEPTS OF ANATOMY AND BODILY PROCESSES}

The concepts of anatomy which form part of the Tabwa medical system include not only the delineation of external body parts, but also the itemization of internal organs and the specification of their functions. For BaTabwa, organs work in relative isolation from one another, and do not constitute structures organized into systems which operate as wholes. In addition, organ function is primarily of significance in discussions of the body as atomized and as in a state of health. With the exceptions of the spleen (safura), whose enlargement causes 'anemia' (kukosa damu), and 'dropsy' (safura), and the heart, whose rapid beating indicates critical illness, BaTabwa do not consider that organs can function pathologically or be subject to attacks by disease. Conversely, the vocabulary of pathology employed in the Tabwa medical system is drawn from the domain of nature, and the human body considered as a whole or thought of in illness can be said to occupy an intellectual space that is in some sense quite different from that occupied by anatomy per se.

The important, normal bodily processes of hunger and gestation result from the actions of the two "snakes" (nyoka) or "insects" (wadudu) which occupy the body of every human being. Though these creatures are essential, they are also capable of pathologic action. It is they who cause certain types of abdominal illness and reproductive difficulties. Others of their kind are responsible for inflammations of the limbs or joints, some of which result in permanent disability. Though the image of the 'insect' BaTabwa effectively conceptualize a series of transformations which are simultaneously systematic in their progression and yet alien to the consciousness and/or wellbeing of the person in whose body they exist.

In other medical settings, particularly those which have to do with the preparation of herbal medicines, strong analogies are made between the body and trees. The upright stance of the one is comparable to the vertical position of the other; feet, torso and head correspond to roots, trunk and branches respectively; and the runners or roots by which some plants propagate offshoots are comparable to the umbilicus which is both the materialization of a woman's fertility and the cord uniting one generation of people to the next.

Further, several important deviations from health are directly attributable to the entrance into the body of cold, external winds (pepo). Certain types of dizziness (zungu-zungu) and diarrhea (kuendesha tumbo) are the results of cold winds entering the abdomen via the anus and the fontanels, respectively. Fever (homa) is due to the penetration by the wind of one's pores, a penetration one senses as the chills or coldness (baridi) which precede the onset of the hot stage of the illness.

Finally, digestion and coitus constitute bodily processes whose functions have complementary meanings in Tabwa medical thought. In contrast to other Bantu groups, such as Gikuyu and Zulu, for whom digestion functions as a model of ritual and social transformations $[4,5]$, BaTabwa regard the process as the archetypal reduction of something to nothing. When someone has expended the usefulness of an object or has illegally diverted funds for his own benefit, he says he has "eaten" the thing in question; and this brings to an end others' efforts to retrieve it. Similarly, people often underscore the futility of selfishness by pointing out that food is ultimately nothing more than 'feces in the bush' (mavi mu pori) and therefore not worth denying another. Finally, the latrine is a waste place into which magical medicines and amulets may be thrown, that their powers be irreversibly neutralized.

The amoral, profoundly transforming capacity of coitus stands in sharp contrast to the mundanity of digestion. Uncontained, as in adulterous sexual relations, coitus can cause mortal illness of infants and the death of women in childbirth. Similarly, excessive conjugal intercourse, or intercourse begun too soon after the birth of a child can result in a reversal of the child's normal developmental processes, occasionally leading to death. Coitus must also be isolated from certain "hot" (moto) illnesses, such as "measles" (suruba) and "smallpox" (ndui) and others, such as "lunacy" (wazimu) and "epilepsy" (kifafa), whose progress also must be carefully kept on its most benigin course by means of medicines. In addition, cultural transformations such as beer brewing, potting, and the manufacture of oil from peanuts and/or sesame seeds must be protected by abstinence from coitus if they are to yield satisfactory results.

Finally, coitus that is ceremonially 'framed", as it were, i.e. intercourse that is either interrupted or per formed under special conditions, results in the fabrication of 'new' cultural entities. In their first sexual contact after the birth of a child, a husband and wife perform a ceremony by which the infant is "matured" (kumukomesha). To accomplish this, they practice coitus interruptus with the infant between them. The 
parents than arise with the child, administer to him a warm infusion of herbal fertility medicines and tie around his waist a protective string which has been dipped in these medicines. By means of this procedure, the child's body is closed to disruptive environmental forces, and becomes capable of responding to treatment with medicines fabricated by those who have had sexual relations the night before.

Similarly, in the bupyani ceremony whereby a widow or widower is cleansed of the coldness deriving from the death of the spouse, the transformation is accomplished by ceremonial intercourse between the survivor and a kinsman of the deceased, after which the couple may remain married or separate, as they wish. Without such a ceremony, the survivor may not approach a fire, may not wash with hot water and may not marry again.

With the practice of incest (kisoni), individuals employ ceremonial intercourse to fabricate amulets which will bring them great wealth. Such an amulet is first compounded by a practitioner, then taken home by the client who has intercourse with his sister or daughter in the presence of the object. The amulet is then returned to the practitioner for completion. The use of incest in this way is an act of sorcery.

Coitus, then, is the physiologic analogue of fire. Uncontained by the bounds of marriage and disciplined abstinence, coitus works to the detriment of bodily integrity and of cultural processes involving the use of a controlled amount of heat. In this respect it is comparable to the destructive burning which can be the result of a fire that is out of control.

Inversely, by appropriate control and containment, the power of coitus can be prevented from the shortcircuiting of other, more delicate cultural transformations, that these come to successful conclusion. Beyond containment, it can, as ceremonial intercourse, have its power directed toward purely cultural ends. Similarly, fire's capacity to burn can be controlled through the use of objects such as pots and media such as water or oil, that its transformative capacity be fully directed toward the fabrication of new entities. Connecting the two affecting powers is the warmed water set out in the evening by a woman for her husband when she desires him, and the warmed water that it is a husband's prerogative to receive every morning upon arising.

In the biosocial domain, as well, there is a structure of relations comparable to that which obtains for fire and coitus. Health is an unmarked, balanced state in which an individual "feels nothing" (hasikii kitu) other than hunger. The apertures and boundaries of his body are closed to alien environmental forces, and he suffers no excess of either cold (as chills [baridi]) or of heat (as fever [homa]). With illness, the situation is reversed. Bodily integrity deteriorates as symptoms become ascendant. With the ending of life in critical illness, the body becomes cold and and the blood stops circulating, while the pulse becomes rapid and weak. Death is an extinguishing (kuzimisha) of life, and those who have been tainted by it must refrain from contact with fire or with hot water until they have been appropriately cleansed.

Life, coitus, and fire are thus transformative powers which are identified with one another in very important ways. They take the shapes of entities but are really processes whose materializations as the body semen, and the flame, are not the static, reified objects they might seem to be. Instead, each contains a constant potential for transformation and self-transformation, making the unmarked or steady state a fundamentally dynamic condition.

The manifest continuity of the imagery of bodily events with that of natural occurrences has a similar effect. What goes on inside a human being is not different in kind from transformations unfolding without.

Hence, one can meditate on the ordered processes of nature, and thus come to know something of physiology. Inversely, one can meditate upon bodily events and come to know something of the world.

\section{DIAGNOSTIC CATEGORIES AND DIVINATION}

The Tabwa medical system provides some 203 diagnostic categories in terms of which specific instances of illness can be described and classified. Of the total, approximately 26 can be distinguished as simple categories, corresponding superficially to the 'symptom' of the Western biomedical system, and referring exclusively to a single, physiologic deviation from health (e.g. coughing, fever, chills, nausea, etc.). The presence of any one of these is sufficient to constitute an instance of illness; and inversely. deviations from a normal state which do not fall into one of these categories are not instances of being sick, but are "conditions" (hali) (c.g. blindncss, crippledness, pregnancy).

Simple diagnostic categories can be combined and recombined as necessary, to generate a complete description of a given illness occurrence. For this reason, they tend to form the pool from which presenting complaints are drawn. Finally, simple diagnostic categories make no reference to temporal sequence. Symptoms are either present or absent, and there is no neccessary order to their successive appearances.

In contrast to these stand complex diagnostic categories. These superficially correspond to the 'syndromes' of Western biomedicine, but include in their composition features of the illness drawn from domains other than the exclusively physiologic.

A complex category may include reference to such features as the age and sex of the patient, his activities immediately prior to the onset of the occurrence, the pathology of the illness, its prognosis, and the alternate forms its progress may take. Where simple diagnostic categories appear to be the terms in which illness is given shape. terms which can be applied by anyone, the classification of a given instance of illness into a complex category is often a matter of some speculation and requires an expert opinion. Similarly, complex categories are not generative, and cannot be used to create 'new' descriptions of illness. Rather, an occurrence which does not display all the features of a given category may fall 'between' categories, and be difficult to diagnose as a result.

The pattern established in Tabwa concepts of anatomy and bodily processes is thus continued here. Just as events unfolding within the body were identified with events occurring without, so do the very 
terms in which disease is conceptualized function to wed the physiologic to the social circumstances and characterics of the patient. Beyond even this, as well, is the problematic illness for which divination is sought, an occurrence such as the one affecting Kaputa's life.

A problematic illness is an occurrence of any kind which displays one or more of three characteristics:

(a) it threatens the patient with permanent disability or death:

(b) it is unintelligible (i.e. it falls 'between' diagnostic categories); and

(c) it is unresponsive to treatment.

Any of these features is sufficient to prompt questioning as to the etiology of the occurrence, and, for BaTabwa ultimate causes lie not in his physiology but in the patient's social-historical circumstances. Divination is the only means by which etiology can be known, and it is regularly sought, as with Malaika's convulsions and fever, when an illness is life-threatening. Illnesses which are merely unintelligible and/or unresponsive to treatment, such as the swellings on the back of Maliaka's head, do not require immediate investigation, though eventually some effort at deciphering them should be made.

When they have decided to divine, people have before them several alternatives of varying accuracy and expense. Regardless of which one they choose, the procedure by which the investigation is set in motion is the same: there is first an invocation (kulandila) of the 'arrow' in which the cause of the illness is commanded to present itself to the diviner, which is then followed by a seance in which a diagnosis is presented. As we have observed in Kaputa's case, the diviner's hypothesis ties together the nature of the illness (both physiologic and social), the history and circumstances of the patient and his kin, and one of the four different etiologic agents that are responsible for all problematic illness occurrences.

Of importance to this discussion is the profound communicative significance accorded to the unique event by the structure of the divinatory or oracular system. This significance has two domains: that of the problematic illness itself and that of the means by which it is deciphered or recognized.

First, the problematic illness, as described above, is an atypical or unique event, defined in the Tabwa medical system as the embodied and muffled communication of an etiologic agent such as an ancestor (mzimu), a spirit (pepo), an avenging ghost (kibanda), or sorcerer (mlozi). In this capacity, problematic illness has the privilege of precipitating what $I$ have elsewhere called a "moment of concluding", a period in which the patient and his kin meditate upon the combination of circumstances and meanings which together constitute an individual's personal identity, i.e. what he knows to be true of himself. In this developmental restructuring of the self, the process of divination and the discursive authority of the diviner (i.e. his right to define the patient's circumstances) both play important roles.

For BaTabwa, then, problematic illness is only superficially a punishment or an attack. At a more profound level it is, in its uniqueness, the indicator and potential revealer of significant truth. It is a mess- age that is 'unintelligible' (haijulikani) because it is said in the symptoms afflicting the body. When the message is made explicit in the diviner's words, the nonce communication unfolding in the body can cease and the patient begin to show signs of improvement even without further modifications of his physiologic therapy.

On the second level, that of divination itself, the unique event takes the form of the coincidence. Not only are the plausibility and elegance of the diviner's hypothesis determined by the extent to which he effectively correlates circumstances occurring in three different domains (the physiologic, the social, and the spiritual), but the very hypothesis itself is often revealed and/or validated by the coincidence that is the voice of the oracle.

Whether it be the diviner's dreams-whose contents are governed by the instructions made during the preparation of the 'arrow'-or the throwing of divinatory bones or objects, or the brewing of oracular beer, the converging epistemologic implications of the process are both clear and deep. First. the communicative significance of the unique event proposes a relation between the hidden and the manifest which is such that knowledge of this relation can be obtained and validated without the intervention of mathesis (i.e. a mathematically based evaluative grid). Second, the definition of coincidence as an event of special discursive fullness gives particular epistemologic emphasis to the witnessing eye. BaTabwa make the point more succinctly when they say that a diviner is like the laboratory technician at a clinic. Just as the technician examines one's blood, urine and feces under the microscope and sees there parasites not visible to the naked eye, so does the diviner employ his special vision to see into one's affairs and discover there causes of jllness to which a lay person would be blind.

The two optical images are important for our understanding. A microscope magnifies structures directly, rendering them accessible to vision and comprehension while maintaining their sanctity and uniqueness. So does the oracle in the divination cut directly to the heart of the problem, transcending the limits of normal insight and defining the particular illness occurrence without reference to an intervening network of crosscutting, statistically determined typical cases.

In addition, BaTabwa consider that the diviner's special power inheres in his vision (mumacho yake) Practitioners are said to 'have eyes' (kuwa na macho) which enable them to see what others do not. Lay people, in contrast, are 'blind' (kipofu). They go about 'like children' (kama vile watoto), with only the most superficial understanding of the nature of events. It is in his capacity as witness that the diviner is able to put into a meaningful whole circumstances and happenings which might remain unconnected, but for the capacity which lies at the center of his forehead and governs the way he sees.

What is being said here is that reality has a fluidity and an interactive quality that accords to the eye a status at once profound and powerful, for it is only under the gaze that an event assumes it truest nature This nature derives from hidden 'meaning' which for all its relative obscurity is nonetheless of the same 
order as the manifest events themselves, and takes as its shape such things as human motives and social rules.

\section{RELIGIOUS AND MAGICAL PROCESSES}

If the diviner's special vision enables him to witness the connection between the hidden and the manifest, his transformative powers enable him to manipulate it. For each of the four etiologic agents which may cause problematic illness there is an appropriate nonbodily therapy -a therapy which is directed not toward the patient's physical person, but toward the social relations in which he exists. The restoration of order in social relations which have been disrupted, and the realization (i.e. manifestation) in social life of the meanings which previously have been obscured within it are both processes which are essential to the patient's recovery. The four types of ceremony accomplish their aims in two complementary ways.

Ceremonies performed on behalf of ancestors (mizimu) and spirits (pepo) derive their particular shape from the nature of the beings with which they are concerned. Ancestors and spirits are those whose intent is the meaning hidden within a given situation. It is they whose will governs the ultimate outcome of the unfolding events, whether good or ill. A principal aim of ceremonies relating to them is the containment of their transformative power and its translation into words. Both of these are processes essential to the restoration of appropriatc communications between these beings and men.

With mizimu the containment is accomplished through the establishment of a small shrine. The process is initiated by the brewing of ordinary corn and millet beer (kibuku) which, in this instance, is transformed into a communicative device by means of an invocation (kutambikia). At the outset of the brewing, the ancestor is called upon to express its will through the medium of the beer. A potent drink indicates that it is, indeed, the ancestor named who is responsible for this illness and that this being is willing to enter into special relations with the patient. Beer which spoils constitutes a negative response and necessitates another divination.

Similarly, with pepo--possessing spirits whose arrival in Tabwaland dates from around the thirties--the diviner's hypothesis must be confirmed by the spirit itself. At a ceremony called 'arranging the spirit' (kutengeneza pepo), the afflicted individual (or his representative) is 'mounted' by the pepo, which then speaks through him to state its name and to specify the benefits it has come to confer.

What occurs in both these cases is the use of a material medium (the beer and the body) to realize an intention which is not otherwise directly or immediately accessible. Whether passively or actively, the will of the ancestor or of the spirit is given verbal articulation by means of this medium and is thus both made manifest in and contained by the wider social situation in which it was previously disruptive. If the communicative/transformative structure employed in these ceremonies is effective because it provides a vehicle by which the hidden can be made real, the structures employed to govern the two deadly types of etiologic agent are effective because they obscure what is manifest.

As we saw in Kaputa's case, a potentially fatal connection to an avenging ghost can be severcd by a ceremony called "throwing the person in the bush". In this, the patient and his family go to the bush at night and in silence. There they meet the diviner who washes them with medicines which function to convince the ghost that it has already killed its intended victims and that the people leaving the site are 'nothing' (bure) to it.

When an illness is caused by sorcery the connection between the sorcerer and his victim can sometimes be severed by the fabrication of an amulet (erisi). Called mwanzambale, the amulet is composed of plant and animal substances (miti and tizimba, respectively) and functions to protect the patient in several ways. It transforms the patient's appearance in the eyes of those who would attack him, making them see him kindly or with indifference. It also conceals the patient from those who came to harm him and prevents their magical medicines from crossing his path. Finally, it also reflects back to the sender whatever misfortune he may project toward the patient.

With avenging ghosts and sorcerers, then, the material medium (i.e. the medicinal infusion and the amulet) is a realization of the intentions of men. Activated through invocations (kusemelea) spoken over them by the practitioner, such media both deploy human will in a social setting and give to that will proximate power over the wider situation. The power bestowed by amulets and infusions derives from changes they effect in the eye of the beholder. Threatening beings are held at bay by a process in which the manifest is made obscure.

Underlying and defining the effectiveness of these two types of non-bodily therapy (i.e. the religious and the magical) is the complex interconnection uniting words, objects and events. It is by means of this that the relationship between the hidden and the manifest can be manipulated and the circumstances of the patient's life be gradually shaped to a given end.

In Tabwa thought, the power of words lies in their ability to processualize reality. In the single moment of their utterance, words unite past and future, tying them together with articulations of causality and of intention. In so doing, speech, like life, fire and coitus, causes a thing to mature (kukomesha), to make manifest within itself the process by which it attains the fullness of its being. Speech is associated with continuity and with the east, the propitious direction from which the sun rises, and the dead return t $\rho$ be reborn. It is also associated with culture, with human consciousness, and with the transcendence of becoming over being. Like light, into which the voice (sauti) of a person is transformed at his death, speech makes things and people into what they really are.

Yet for all their ability to make simultaneously accessible past, present and future, words are highly ephemeral. Their totality exists only in the moment of their saying; and to objects falls the task of synchronizing their temporality--in the case of religion, or of temporalizing their synchrony - in the case of magic.

Thus, through the medium of beer or of the body, objects make manifest the will of ancestors and spirits which might otherwise be known only by its un- 
mediated, instantaneous effect upon unfolding events For ancestors and spirits, according to Tabwa thought, act directly. Their words are deeds.

Inversely, as magical infusions and amulets, objects function to preserve the worlds generated in the images of diviners" invocations. Such statements cause the amulets 'to mature' (kukomaa), and become tiny microcosms (kadunia) of the world. Thus activated, they maintain the vitality of the client's intentions, while his thought returns, as it inevitably must, to the more pressing and pragmatic circumstances of ordinary life. In this way do amulets form things like holes in time, through which can be seen the clear intent of practitioner and client, however long ago it may have been expressed, and however obscured it may have been made by the force of intervening events.

Events, for their part, are diachrony itself. Composed as they are of the interweaving of meaning and circumstance, of intention and chance, they are the embodiment of history. In them are hidden processes first obscurely indicated, then clearly revealed. In them as well can one decipher the ultimate will of the ancestors, and determine the extent to which one's own proximate will shall prevail.

The three entities of this transformative complex words, objects and events -can thus be said to be variations of one another. What can be expressed or created in one can be brought into heing in another, yet each has an entirely different constitution with respect to time, materiality and space. In the nonbodily therapies which are crucial to the Tabwa medical system, transformations of events are attempted by aligning in the desired way words and objects. If one does this, BaTabwa say, and God is willing, the circumstances themselves will also conform to the pattern.

\section{CONCLUSION}

This discussion of the Tabwa medical system was begun with the description of a particular case. In it recourse was made to both European and traditional medicines, and to the process of divination. Subscquent consideration was devoted to the exposition of principles which were present, though tacit, in the case as given; and which are deeply rooted in Tabwa concepts, not only of illness and health, but also of anatomy, bodily processes and non-bodily transformations. Kaputa's decisions about his daughter were thus placed against a background of widening circles of comprehension. In this conclusion there will be a discussion of some of the widest domains of conceptualization, and then a return to the center, to the case, to accurately locate Western medicine within a total epistemologic context.

In our consideration of the principles which underlie the theory and practice of medicine for BaTabwa, we have been led to concepts of the transposibility of symptoms, events and words; to ideas about transformation, and to thoughts about the relationship between the material and the meaningful in the unfolding of events. Beyond all of these are Tabwa ideas about time and about change, as these relate to the body and to health and illness.
Despite the presence and extensive use of clocks, calendars and radios, there is an important respect in which time is not measured by BaTabwa in terms of minutes, hours and days ticking off at a fixed and relentless rate. In an environment where mechanization has not made overwhelming inroads into the conceptualization of life, time may be defined as a sequence of coincident processes, all of which give way to one another in a regular and regularly moving order. Thus it is that the smoke of the dry season's bush fires rises to the sky where, people say, it forms the clouds which bring the rains of October. So, too, does the moon grow fatter and fuller as it moves from west to east, only to wane there, before returning, slender and rejuvenated to the west. Human beings participate, as well, in this process. One is born. matures and dies only to be reborn to a kinsman, or to be 'rejuvenated' in the memory of oneself that is an infant namesake.

Within this broader unfolding also occurs the enacted or embodied time of which individual experience is composed. Its progress can be measured by the gradual clearing of a substantial field, by births of one's children, and by major events such as the deaths of one's close kin. In such a setting one is perhaps less in time than time is in oneself; identified with change, time comes into being in large measure by means of one's own activities and efforts, even when these are coordinated within an agricultural cycle. Such is the case at least when one is healthy and one's determination is one's action.

In illness this relationship of experience to time is disrupted, indeed, is reversed. The passage of time is 'felt' by one whose illness divides his will from his ability, and leaves him 'without self-nastery' (hajiwezi), as people emphasize when speaking of serious illness. Time is doubled, as household members sit up all night (kesha) watching by the side of a sick kinsman, and determining the hour by the position of the Milky Way (kipinda busiku or "that which turns the night"). People will often say that someone was ill for three days, then correct themselves to indicate that it was really six hecause it was three days and three nights.

Further, illness brings to bear upon the family of the patient the pressure of time speeded up. Instead of basing their choices upon their own necessities and convenience, people must move to the pace set by the progress of the disease. Procrastination can result in an illness which has become so serious that no practitioner would be willing to treat it, and it was with the danger of their daughter's fever in mind that Kaputa and Kalwa moved rapidly to seek divination.

In a situation such as this, then, time is change, and the body-or the material substance of a thing--is but the artifact of the processes that are time unfolding, blossom-like, from within it. In health. these go all unnoticed, but in illness, process becomes identified with deterioration and time becomes the product of the 'Other' that governs the progress of the disease.

Modification can be effected from without. as when non-bodily therapy changes the context in which a patient lives as well as from within by means of traditional and European medicines; and it is here, in the relatively restricted domain of physiological therapies that BaTabwa distinguish between davia la 
kizungu (European medicines) and ya kinchi (medicines of the land).

Each of the two has a distinctive form. Pills, injections and liquid medicines characterize the first, while the latter comprises plant and animal substances derived from the bush. Though its principal loci are the dispensary and the hospital, European medicine may also be obtained for itinerant merchants and fellow villagers, as was the niridazole that Kaputa purchased. The Tabwa medical system includes a body of lay knowledge governing the use of European medicines, and this is known to virtually every adult in much the same way as every adult knows at least some of the traditional medicines that are readily available in the area.

While each type of medicine has its strengths and its weaknesses, what is more to the point of this discussion is the fact that both are subordinate in the same way to the broader epistemologic concerns that give the Tabwa medical system as a whole its meaning and vitality. Thus it is that Kaputa, his wife and his family could freely alternate between traditional and European medicines and never feel that the utilization of the latter necessitated the entry into an epistemologic system whose underlying principles were fundamentally different from their own. Instead, their approach to Malaika's illness combined a pragmatism which would permit the use of any physiologic medicine that would work with a meditative concern that sought to structure the divination in such a way as to obtain the most veracious insights possible.

In his essay on the form and meaning of magical acts, Tambiah suggests that "it is fundamentally mistaken to say that African religion and ritual are concerned with the same intellectual tasks that science in Western society is concerned with" [6]. While successful treatment is measured in both Europe and Africa by the effectiveness with which symptoms are made to remit, our discussion of the principles underlying the Tabwa medical system would indicate that success in it means something entirely different from success in a Western hospital. The Tabwa system, for all its use of European medicines, is essentially directed toward the alleviation of suffering as a fact of human experience. If it accomplishes this task by causing the remission of symptoms it is doubly successful; but the totality of its concerns is such that the persistence of symptoms is no measure of its failure.

Any medical system must fail to heal each of its constituents at least once in his life; hence, as important as the restoration of physical wholeness is the elaboration of a system of meaning, partly personal and partly cultural, which can expand to encompass the vicissitudes we experience and thus make sense of them. Though remission of symptoms remains the ultimate confirmation of a diagnosis, for BaTabwa "to recognize the illness" (kutambuwa ujonguwa) is to understand and speak about more than physiologic circumstances.

In the Tabwa medical system, the body holds the position of a defile or conduit through which flow and are united the mass of events that are the raw material of a patient's history and the several etiologic agents which serve as anchoring points, giving structure to the flow of circumstance and transforming it into meaningful narrative. This is the task to which the Tabwa medical system rises and toward the fulfillment of which it directs even the use of European medicines.

\section{REFERENCES}

1. The material presented in this paper derives from four years" field research (1974-1977) undertaken among the Lakeside Tabwa of Shaba, Zaire. The Tabwa are a matrilineal virilocal people who live primarily by farming manioc and fishing the rich waters of Lake Tanganyika. They are culturally similar to their southern neighbors the Bemba, but share other cultural characteristics with their neighbors to the north, the Luba. Funds for the study were derived from a U.S Public Health Service Training Grant, a Wenner-Gren Grant-in-aid, and a Social Science Research Council Foreign Area Fellowship. Opinions expressed in the paper are those of the author.

2. Words enclosed in single quotes are English glosses of Swahili terms. In the village of Mpala, as well as in a substantial area surrounding it. Swahili has become the primary language, having been introduced nearly a century ago by Kingwana traders and European explorers. Though it is understood by the majority of the adult population, KiTabwa is not spoken by any but the most elderly or those born in the interior. Children born since independence speak and understand only Swahili. The culture described here appears to be a modification of the traditional system, changed and individualized by a coherent process of transformation into one better suited to the demands of present-day life.

3. The shore around Mpala is both rocky and steep. This effectively prevents the growth of the grasses eaten by the snails which are the intermediate hosts of the blood flukes that cause bilharziasis. As a result, the only cases of the disease found in the ared are those contracted elsewhere.

4. Kenyatta J. Facing Mt Kenya: The Trihal Life of the GiKuyu. Vintage, New York, 1937

5. Ngubane H. Body and Mind in Zulu Medicine. Academic Press, New York, 1977.

6. Tambiah S. J. Form and meaning in magical acts: a point of view. In Modes of Thought: Essays on Think ing in Western and Non-Western Societies (Edited by Horton R. and Finnegan R.), p. 225. Faber \& Faber London, 1973. 